\title{
Deep echo state network with reservoirs of multiple activation functions for time-series prediction
}

\author{
YONGBO LIAO ${ }^{1,2, *}$ and HONGMEI LI ${ }^{1}$ \\ ${ }^{1}$ School of Electronic Science and Engineering, University of Electronic Science and Technology of China, \\ Chengdu, China \\ ${ }^{2}$ State Key Laboratory of Electronic Thin Films and Integrated Devices, University of Electronic Science and \\ Technology of China, Chengdu, China \\ e-mail: 1yb@uestc.edu.cn; 1hm3551@qq.com
}

MS received 3 July 2018; revised 30 September 2018; accepted 7 March 2019; published online 22 May 2019

\begin{abstract}
In this paper, an improved deep echo state network is proposed, named as multiple activation functions deep echo state network (MAF-DESN), where states are activated by multiple activation functions. A sufficient condition for MAF-DESN is given to guarantee that MAF-DESN possesses the echo state property. Finally, the MAF-DESN is applied to chaotic time-series predictions and compared to other ESN deformation models and popular LSTM. Simulation results show that under same network size condition, MAF-DESN possesses stronger explanatory power in chaotic far-infrared laser predictions $(R$-square $=0.9537$, others $\leq 0.6487$ ), and better fitting ability in daily foreign exchange rates (MAE $=0.0040$, others $\geq 0.0047$ ) and chaotic far-infrared laser (MAE $=3.4042$, others $\geq 4.9021)$. In high-dimension-input task, MAF-DESN improved the performance when the results were compared $(R$-square $=0.4274$, others $\leq 0.3975$ and $\mathrm{MAE}=5.2221$, others $\geq 7.6876$ ), while the train time of MAF-DESN did not increase when compared to DESN.
\end{abstract}

Keywords. Deep echo state network; reservoir computing; multiple activation functions; time-series prediction.

\section{Introduction}

In machine learning and computational intelligence communities, an increasing number of neural network models, especially echo state network (ESN), are being applied to time-series prediction tasks. Over the last decades, the classic ESN has been recognized as the most efficient solution for training recurrent neural network (RNN), possessing many merits such as simplified training process and less time consumption. However, the performance of ESN suffers due to the inherent ill-posed problem. For the sake of refining the classic ESN, researchers attempted many optimizing approaches to improve the performance of ESN. Optimizing approaches of ESN are divided into two groups approximately. The first group of optimizing approaches vary the details of original ESN, such as varying gain and bias of activation function [1], replacing normalized root mean square error (NRMSE) by correntropy [2, 3] and adding Laplacian algorithm [4] to reduce the dimension of reservoir states. The second group of optimizing approaches mainly combine the ESN with other complementary neural networks or methods, including but not limited to echo state queueing networks (ESQN) [5],

*For correspondence robust echo state networks (RESN) [2], combination structure of reservoir computing and support vector machines (RCSVM) [6], deep belief echo state network (DBESN) [7], deep recurrent neural network (DRNN) [8], optimized echo state network (OESN) by binary particle swarm optimal algorithm [9], stacked denoising autoencoders (SDA) [10], dynamical regularized echo state network (DRESN) [11], adaptive lasso echo state network (ALESN) [12], recursive Bayesian echo state network (RBESN) [13], polynomial echo state network [14], multilayered echo state network (MLESN) [15] and stacked deep echo state network (DESN) [16].

Aforementioned DESN is similar to MLESN, which is a deep structure composed of multiple reservoirs. Like other deep structures [17-28], DESN is a network with more than three layers, and has been applied to multiple simulation tasks [15]. However, to our best knowledge, there is little literature about sufficient condition of DESN and directly applying the sufficient condition of ESN is not suitable since the input weights of hidden layer to layer are similar to reservoir weights of ESN, which also need constraints. Hence, we propose the sufficient condition of DESN as well as the calculation process in the main body to guarantee convergence. However, in traditional ESN or DESN, the hidden neurons are activated by a single 
activation function (mostly tanh function). Due to different optimized weighted parameters corresponding to different learning tasks, a linear combination of activation functions is more reasonable than a single activation function [29]. Hence, we propose multiple activation functions DESN (MAF-DESN) to explore the suitable activation function combination that makes the update process of reservoir states of hidden layers more reasonable.

The main contributions of this paper are as follows. 1) DESN is a novel deep structure, and there is little literature on its sufficient condition that guarantees the convergence of reservoir update process. In this paper, we follow the convergence condition proposed in [30] and propose the convergence sufficient condition in section 2 with the calculation process in Appendix A. 2) DESN is activated by a single activation function, which is fixed and unalterable in the training process. In this paper, we use more than one activation functions with different forms to replace the original single activation function, whose coefficients are unfixed and alterable. Considering a linear combination of activation functions as a combined activation function, the combined activation function is changeable when coefficients change. In the MAF-DESN, each reservoir shares the same activation functions layer to layer with changeable linear combination coefficients, which is novel and worth studying. 3) Dealing with time-series tasks is an advantage of DESN. In our experiments, we apply MAF-DESN to multiple chaotic time-series predictions, including highdimension-input task. On comparing DESN with single activation function, the prediction accuracy is improved.

The remaining part of this paper is organized as follows. In section 2, we review the theories of the DESN and propose the convergence sufficient condition. In section 3, we briefly introduce the novel MAF-DESN and its sufficient condition and prove it in detail in Appendix B. Experiments and analyses are performed in section 4 . The discussion and conclusions of this paper are given in section 5 .

\section{DESN}

DESN, as illustrated in figure 1, is a novel network, which replaces the hidden layer of ESN with multiple reservoirs.

It comprises an input layer, which loads outside data into network, multiple reservoirs, which conduct data processing progress, and an output layer gathering hidden layers

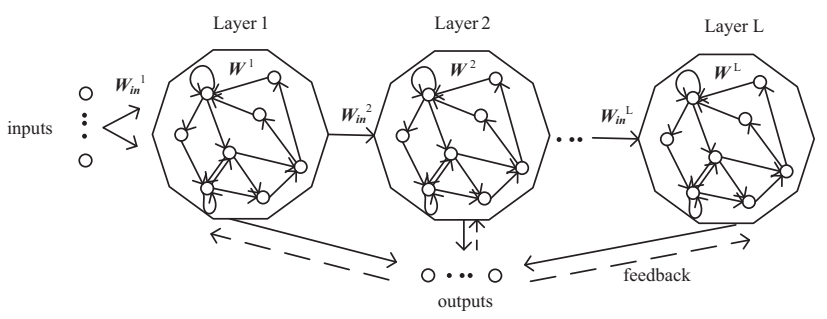

Figure 1. Deep echo state network structure. states to calculate network prediction results. The number of inputs, reservoir neurons of layer $1, \ldots$, reservoir neurons of layer $L$, and output neurons are $K, N_{1}, \ldots, N_{L}$ and $P$, respectively. Let $\boldsymbol{u}(n)=\left[\begin{array}{lll}u_{1}(n) & \cdots & u_{k}(n)\end{array}\right]^{T}$ denote external input at time $n, \boldsymbol{x}^{(l)}(n)=\left[\begin{array}{lll}x_{1}(n) & \cdots & x_{N_{l}}(n)\end{array}\right]^{T}$ denote reservoir states of layer $l(l=1,2, \cdots, L)$ and $\boldsymbol{y}(n)=\left[\begin{array}{lll}y_{1}(n) & \cdots & y_{P}(n)\end{array}\right]^{T}$ denote output vector. $\boldsymbol{W}_{i n}^{(l)}$, $\boldsymbol{W}^{(l)}$ and $\boldsymbol{b}^{(l)}$ denote weight matrix of input, reservoir and bias of layer $l(l=1,2, \cdots, L)$, respectively, and $\boldsymbol{W}^{\text {out }}$ denotes weight matrix of output, and the sizes of the matrices are $N_{1} \times K(l=1), N_{l} \times N_{l-1}(l=2, \cdots, L), N_{l} \times$ $N_{l}(l=1, \quad 2, \cdots, L), \quad N_{l} \times 1(l=1,2, \cdots, L) \quad$ and $P \times\left(N_{1}+\cdots+N_{L}\right)$. The discrete equation of the DESN model is given as follows [16]:

$$
\begin{gathered}
\boldsymbol{x}_{\boldsymbol{i n}}^{(l)}(n+1)=\boldsymbol{W}_{\boldsymbol{i n}}^{(l)} \boldsymbol{i}^{(l)}(n+1)+\boldsymbol{W}^{(l)} \boldsymbol{x}^{(l)}(n)+\boldsymbol{b}^{(l)} \\
\boldsymbol{i}^{(l)}(n+1)=\left\{\begin{array}{l}
\boldsymbol{u}(n+1), l=1 \\
\boldsymbol{x}^{(l-1)}(n+1), l>1
\end{array}\right. \\
\boldsymbol{x}^{(l)}(n+1)=\left(1-a^{(l)}\right) \boldsymbol{x}^{(l)}(n)+a^{(l)} f^{(l)}\left(\boldsymbol{x}_{\boldsymbol{i n}}^{(l)}(n+1)\right) \\
\boldsymbol{y}(n)=g\left(\boldsymbol{W}^{\text {out }} \boldsymbol{x}(n)\right)
\end{gathered}
$$

where $n$ is index time, superscript $l$ denotes layer, $\boldsymbol{i}^{(l)}(n+1)$ denotes the input signals of $l$ th layer, $\boldsymbol{x}_{\boldsymbol{i n}}^{(l)}(n+1)$ denotes the weighted input data of reservoir neuron of $l$ th layer, $f^{(l)}$ denotes a sigmoid function inside reservoir of $l$ th layer (usually tanh function or logistic sigmoid function) and $a^{(l)}$ is leaky rate of $l$ th layer limited $\operatorname{in}(0,1]$. When $a^{(l)}=1$, Eq. (3) can be simplified as

$$
\boldsymbol{x}^{(l)}(n+1)=f^{(l)}\left(\boldsymbol{x}_{\boldsymbol{i n}}^{(l)}(n+1)\right) .
$$

The $\boldsymbol{x}(n)$ in Eq. (4) is a gathering of reservoir states of all layers

$$
\boldsymbol{x}(n)=\left[\begin{array}{lll}
\left(\boldsymbol{x}^{(1)}(n)\right)^{T} & \cdots & \left(\boldsymbol{x}^{(L)}(n)\right)^{T}
\end{array}\right]^{T} .
$$

$\boldsymbol{W}_{\text {in }}^{(l)}, \boldsymbol{W}^{(1)}$ and $\boldsymbol{b}^{(l)}$ are known and unalterable, but $\boldsymbol{W}^{\text {out }}$ is unknown and alterable. $W^{\text {out }}$ needs to be computed by desired network training, where the gathering of echo states $\boldsymbol{x}(n)$ is classified row-wise into a state collection $\boldsymbol{X}$ and the trained output values $\boldsymbol{y}(n)$ corresponding to the $\boldsymbol{x}(n)$ are put row-wise into a vector $\boldsymbol{Y}$. Then, $\boldsymbol{W}^{\text {out }}$ is computed by the following learning equation [30]:

$$
\boldsymbol{W}^{\text {out }}=\boldsymbol{Y} \boldsymbol{X}^{T}\left(\boldsymbol{X} \boldsymbol{X}^{T}+\alpha \boldsymbol{E}\right)^{-1}
$$

where $\alpha$ denotes regularization coefficient, superscript $\mathrm{T}$ denotes matrix transpose and superscript -1 denotes matrix inversion.

The standard of training DESN is to minimize an error $E(\boldsymbol{d}, \boldsymbol{y})$, which is typically an NRMSE, and the formula is as follows [29]: 


$$
E(\boldsymbol{d}, \boldsymbol{y})=\sqrt{\frac{\left\langle\|\boldsymbol{d}-\boldsymbol{y}\|^{2}\right\rangle}{\left\langle\|\boldsymbol{d}-\langle\boldsymbol{d}\rangle\|^{2}\right\rangle}}
$$

where $\boldsymbol{y}$ is the output, $\boldsymbol{d}$ is the teacher output, $\|\cdot\|$ denotes the Euclidean distance and $\langle\cdot\rangle$ denotes the average function.

According to [30], if ESN network possesses echo state property (ESP), then $\left\|\boldsymbol{x}(n+1)-\boldsymbol{x}^{\prime}(n+1)\right\| \leq \| \boldsymbol{x}(n)-$ $\boldsymbol{x}^{\prime}(n) \|(\|\cdot\|$ denotes Euclidean distance) is satisfied, where $\boldsymbol{x}(n+1), \boldsymbol{x}^{\prime}(n+1)$ are the states generated by the same input $\boldsymbol{u}(n+1)$. Generalized to DESN, ESP is described as $\left\|\boldsymbol{x}(n+1)-\boldsymbol{x}^{\prime}(n+1)\right\| \leq\left\|\boldsymbol{x}(n)-\boldsymbol{x}^{\prime}(n)\right\|$ where $\boldsymbol{x}(n+1)$, $\boldsymbol{x}^{\prime}(n+1)$ are the gathering of reservoir states generated by the same input $\boldsymbol{u}(n+1)$. To our best knowledge, this is the first time of ESP of DESN has been proposed as here. According to ESP of DESN, we obtain following inequations:

$$
\left\{\begin{array}{l}
\frac{\left(1-a^{(l)}+a^{(l)} \Lambda^{(l)}\right)^{2}}{1-\left(a^{(l+1)} \Lambda_{\mathrm{in}}^{(l+1)}\right)^{2}} \leq 1, l=1, \cdots L-1 \\
\left(1-a^{(l)}+a^{(l)} \Lambda^{(l)}\right)^{2} \leq 1, l=L
\end{array}\right.
$$

where $\Lambda^{(l)}$ is reservoir spectral radius of $l$ th layer and $\Lambda_{\mathrm{in}}^{(l+1)}$ is input weight matrix spectral radius of $l$ th layer. Proof of Inequation (9) is given in Appendix A.

Since the sufficient condition of one reservoir is $0<\Lambda<1$, this implies that every reservoir of DESN should meet the following inequation:

$$
0<\Lambda^{(l)}<1, l=1, \cdots, L .
$$

Substituting Inequation (10) into Inequation (9), we obtain sufficient condition of DESN as follows:

$$
\left\{\begin{array}{l}
0<\Lambda^{(l)}<1, l=1, \cdots, L-1 \\
1-2 / a^{(l)}<\Lambda^{(l)}<1, l=L \\
\Lambda_{\mathrm{in}}^{(l)} \leq \sqrt{1-\left(1-a^{(l-1)}+a^{(l-1)} \Lambda^{(l-1)}\right)^{2}} / a^{(l)}, l=2, \cdots L
\end{array} .\right.
$$

Now, the sufficient condition of DESN is calculated. The next section addresses modified DESN and its sufficient condition.

\section{The modified DESN}

Based on DESN, to solve different learning tasks, to explore suitable activation function, utilizing the weighted sum of multiple activation functions to take the place of original single activation function in Eq. (1), one can obtain modified hidden layer update equation as follows:

$$
\begin{aligned}
\boldsymbol{x}^{(l)}(n+1)= & \left(1-a^{(l)}\right) \boldsymbol{x}^{(l)}(n) \\
& +\sum_{i=1}^{N_{\lambda}^{l}}\left(\lambda_{i}^{(l)} f_{i}^{(l)}\left(\boldsymbol{x}_{\boldsymbol{i n}}^{(l)}(n+1)\right)\right)
\end{aligned}
$$

where $N_{\lambda}^{l}$ denotes the number of activation functions of $l$ th layer and $\lambda_{i}^{(l)}$ denotes the coefficient of activation function $f_{i}^{(l)}$. In other words, every layer of this modified DESN is a linear superposition of multiple reservoirs.

For the sake of ESP, the sufficient conditions are given as follows:

1. $f_{i}^{(l)}$ is a sigmoid function or a linear function that satisfies $\left\|f_{i}^{(l)}(x)-f_{i}^{(l)}\left(x^{\prime}\right)\right\| \leq\left\|x-x^{\prime}\right\| ;$

2. $0<a^{(l)} \leq 1, l=1, \cdots, L$;

3. $\sum_{i=1}^{N_{\lambda}^{l}}\left|\lambda_{i}^{(l)}\right| \neq 0$;

4. Following sufficient conditions are for aforementioned models, where $\Lambda^{(l)}$ denotes the maximal singular value of $\boldsymbol{W}^{(l)}$ and $\Lambda_{\mathrm{in}}^{(l)}$ denotes the maximal singular value of $\boldsymbol{W}_{\text {in }}^{(l)}(l \geq 2)$ :

$$
\left\{\begin{array}{l}
0<\Lambda^{(l)}<1, l=1, \cdots, L-1 \\
0<\Lambda^{(l)}<a^{(l)} / \sum_{i=1}^{N_{\lambda}^{l}}\left|\lambda_{i}^{(l)}\right|, l=L \\
\Lambda_{\mathrm{in}}^{(l)} \leq \sqrt{1-\left(1-a^{(l-1)}+\sum_{i=1}^{N_{i}^{l-1}}\left|\lambda_{i}^{(l-1)}\right| \Lambda^{(l-1)}\right)^{2}} / a^{(l)} . \\
, l=2, \cdots L
\end{array}\right.
$$

If aforementioned sufficient conditions are satisfied, then discrete models (12) has the ESP. For a proof of sufficient condition, see Appendix B.

According to the ESP sufficient condition, one can obtain the constraints of the coefficients of activation function as follows:

$$
\left\{\begin{array}{l}
\sum_{i=1}^{N_{\lambda}^{L}}\left|\lambda_{i}^{(L)}\right|<a^{(L)} / \Lambda^{(L)}, l=L \\
\sum_{i=1}^{N_{\lambda}^{l}}\left|\lambda_{i}^{(l)}\right|<\left(\sqrt{1-\left(a^{(l+1)} \Lambda_{\mathrm{in}}^{(l+1)}\right)^{2}}+a^{(l)}-1\right) / \Lambda^{(l)} . \\
\quad, l=1, \cdots L-1
\end{array}\right.
$$

When MAF-DESN is a two-layer model, activated by double activation functions, the constraints of the coefficients of activation function are simplified as

$$
\left\{\begin{array}{l}
\left|\lambda_{1}^{(2)}\right|+\left|\lambda_{2}^{(2)}\right|<a^{(2)} / \Lambda^{(2)} \\
\left|\lambda_{1}^{(1)}\right|+\left|\lambda_{2}^{(1)}\right|<\left(\sqrt{1-\left(a^{(2)} \Lambda_{\mathrm{in}}^{(2)}\right)^{2}}+a^{(1)}-1\right) / \Lambda^{(1)}
\end{array}\right.
$$

which are the constraints used in section 4 .

In conclusion, the ESP sufficient condition is a Lipschitz condition to ensure that the reservoir activation function is a linear combination of contractive mapping. 
Table 1. Activation function influence (test score).

\begin{tabular}{cccccccccc}
\hline$f_{1} f_{1}$ & $f_{1} f_{2}$ & $f_{1} f_{3}$ & $f_{1} f_{4}$ & $f_{2} f_{2}$ & $f_{2} f_{3}$ & $f_{2} f_{4}$ & $f_{3} f_{3}$ & $f_{3} f_{4}$ & $f_{4} f_{4}$ \\
\hline 0.0621 & 0.0308 & 0.0479 & 0.0853 & 0.0455 & 0.0619 & 0.0559 & 0.0813 & 0.0530 & 0.0957 \\
\hline
\end{tabular}

\section{Simulation and analysis}

\subsection{MAF-DESN model experimental analysis}

In this section, numerical simulation experiments are conducted to verify the performance of MAF-DESN. In the first simulation, the dataset is downloaded from web (https://datamarket.com/), which is daily foreign exchange rates (German/US) from 31 December 1979 to 31 December 1998 , scaled to range $[0,1]$ by minmax method before feeding into network. These data are processed as the teacher signal pair <input history data, target data $>$, where data at time $t$ are used to predict data at time $t+1$. The dataset contains 4775 samples, divided into 2 parts: 3199 samples for training and 1576 samples for testing.

The activation functions considered here include

$$
\begin{gathered}
f_{1}(x)=\left(e^{x}-e^{-x}\right) /\left(e^{x}+e^{-x}\right) \\
f_{2}(x)=\left(1-e^{-x}\right) /\left(1+e^{-x}\right)
\end{gathered}
$$

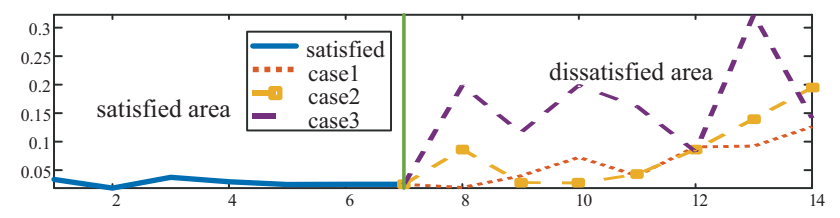

Figure 2. Satisfied vs dissatisfied (activation functions are $f_{1}$ and $f_{2}$, reservoir size is 3 ).

$$
\begin{gathered}
f_{3}(x)=(2 / \pi) \arctan ((2 / \pi) x) \\
f_{4}(x)=x / \sqrt{1+x^{2}}
\end{gathered}
$$

which are nonlinear sigmoid functions satisfying $\left\|f(x)-f\left(x^{\prime}\right)\right\| \leq\left\|x-x^{\prime}\right\|$.

In this simulation, two-layer MAF-DESN is adopted; every layer with two activation functions is updated according to Inequation (12) with $\mathrm{a}^{(l)}=1$, in order to obtain activation functions' influences (table 1), spectral radius with activation function coefficient influence (figure 2) and overfitting problem (figure 3). For sufficient conditions, spectral radius and activation function coefficient must satisfy

$$
\left\{\begin{array}{l}
\left|\lambda_{1}^{(2)}\right|+\left|\lambda_{2}^{(2)}\right|<1 / \Lambda^{(2)} \\
\left|\lambda_{1}^{(1)}\right|+\left|\lambda_{2}^{(1)}\right|<\sqrt{1-\left(\Lambda_{\mathrm{in}}^{(2)}\right)^{2}} / \Lambda^{(1)}
\end{array}\right.
$$

In this simulation, $\Lambda^{(1)}=0.5884, \Lambda^{(2)}=0.5227$ and $\Lambda_{\mathrm{in}}^{(2)}=0.9444$. Hence, sufficient conditions are calculated as

$$
\left\{\begin{array}{l}
\mid \lambda_{1}^{(2)} \\
\lambda_{1}^{(1)}|+|+\left|\begin{array}{c}
\lambda_{2}^{(2)} \\
\lambda_{2}^{(1)}
\end{array}\right|<0.5588
\end{array}\right.
$$

Output evaluation score is calculated by root mean square error (RMSE), namely
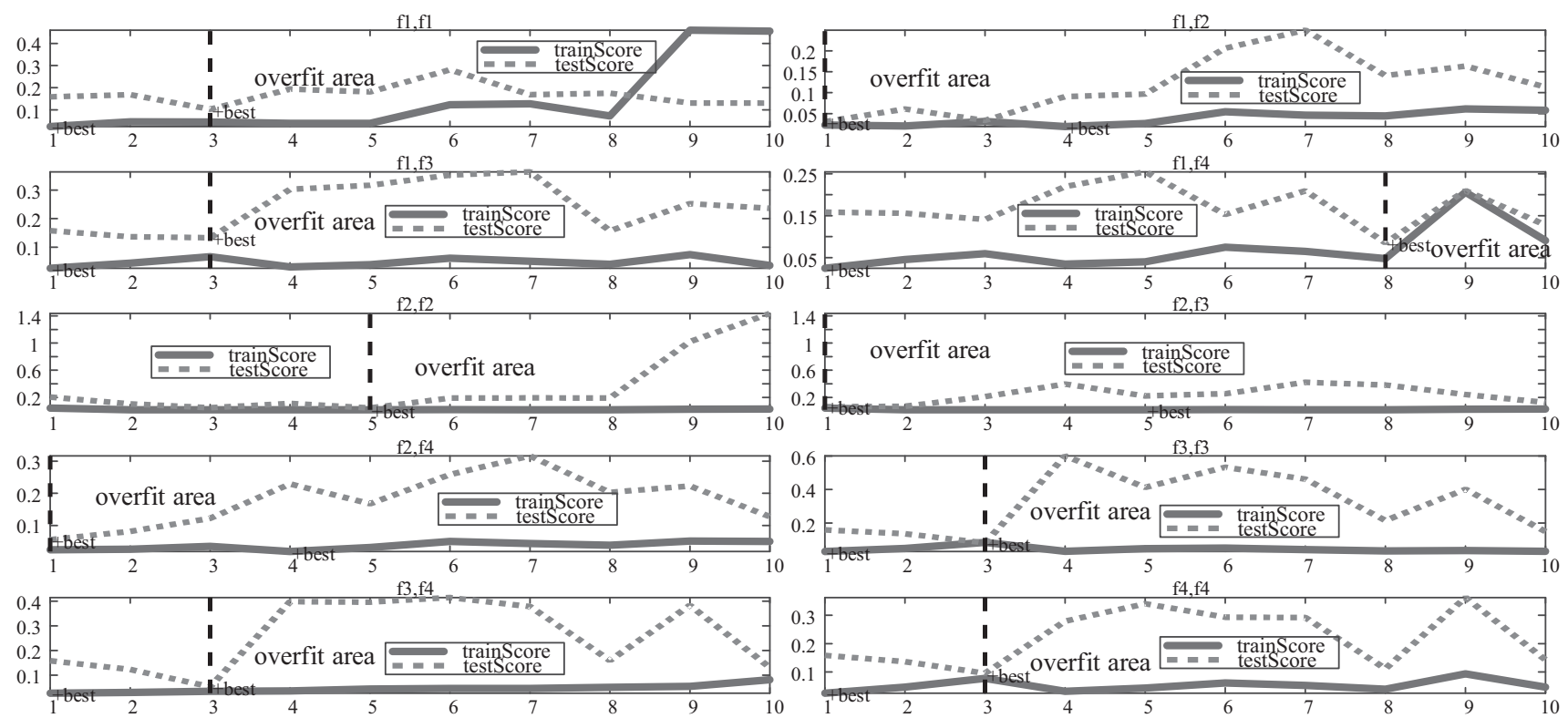

Figure 3. Overfitting curves (when neurons of reservoir increase to 3, test score reaches the minimal RMSE, after which test score increases). 


$$
\operatorname{RMSE}(\boldsymbol{d}, \boldsymbol{y})=\sqrt{(1 / k) \sum_{n=1}^{k}(d(n)-y(n))^{2}}
$$

where $k$ denotes sample size, $d(n)$ denotes target output and $y(n)$ denotes network prediction output.

Table 1 demonstrates that different activation functions slightly influence network training and testing based on same network settings (reservoir size is 3 ). Figure 2 shows the differences of satisfying sufficient condition with dissatisfying sufficient condition (reservoir size is fixed as 3); case 1 denotes that first layer sufficient condition is violated and second layer sufficient condition is satisfied; case 2 denotes that first layer sufficient condition is adhered and second layer sufficient condition is violated; case 3 denotes that first layer and second layer sufficient conditions are violated. When away from the sufficient condition constraint, test score tends to be higher and performance of MAF-DESN tends to be worse. Figure 3 is an example of overfitting problem, where reservoir neurons increase from one to ten. Before reservoir size reaches three, test score gradually approaches training score. When reservoir size is bigger than three, test score gradually gets bigger and drifts away from training score, which implies that MAF-DESN model is over-trained. When neuron size is beyond eight, train score gets bigger, which might be because the higher dimensional space of one-dimensional input map might have upper dimensional limit; in other words, the state matrix is ill-posed. In next simulations, test score is close to train score to avoid overfit problem.

\subsection{MAF-DESN vs other models}

In this section, MAF-DESN model is compered to ESN, ESN-IP [1], ESN-PSO [9], DESN [16] and LSTM.
Evaluation methods are $R$-square (coefficient of determination, closer to 1 denotes stronger explanatory power of the model to output), $R$ (correlation coefficient), RMSE (root mean square error), SSE (the sum of squares due to error) and MAE (mean absolute error):

$$
\begin{gathered}
\mathrm{r}(\boldsymbol{d}, \boldsymbol{y})=\operatorname{cov}(\boldsymbol{d}, \boldsymbol{y}) /\left(\sigma_{\boldsymbol{d}} \sigma_{\boldsymbol{y}}\right) \\
\operatorname{SSE}(\boldsymbol{d}, \boldsymbol{y})=\sum_{n=1}^{k}(d(n)-y(n))^{2} \\
\operatorname{MAE}(\boldsymbol{d}, \boldsymbol{y})=(1 / k) \sum_{n=1}^{k}|d(n)-y(n)|
\end{gathered}
$$

where RMSE, SSE and MAE closer to zero denote better fitting ability. Prediction tasks are samples of daily foreign exchange rates (German/US) (https://datamarket.com/), IBM common stock closing prices (https://datamarket.com/ ) and chaotic far-infrared laser (http://www.weigend.com/). These data are processed as the teacher signal pair <input history data, target data $>$, where data at time $t$ are used to predict data at time $t+1$. In these one-dimension-input tasks, model size is 3, and train sample rate is 0.67. Twolayer MAF-DESN is activated by a combination of $f_{1}$ and $f_{2}$ with best possible guess coefficients, ESN and its variants are activated by $f_{1}$, and LSTM is optimized by Adam optimizer.

From tables 2, 3 and 4, $R$-square of MAF-DESN is closer to 1 than those of other models; meanwhile, SSE, MAE and RMSE of MAF-DESN are closer to 0 than those of other models with similar time consumption, which indicates that the performance of MAF-DESN is better than those of other models considered here. From figures 4-7, peak region (black frame) of MAF-DESN is better fitted than

\begin{tabular}{|c|c|c|c|c|c|c|}
\hline & ESN & ESN-IP & ESN-PSO & LSTM & DESN & MAF-DESN \\
\hline$R$-square & 0.9902 & 0.9906 & 0.9898 & 0.9938 & 0.9866 & 0.9900 \\
\hline$R$ & 0.9951 & 0.9953 & 0.9949 & 0.9969 & 0.9933 & 0.9950 \\
\hline SSE & 0.6823 & 2.6878 & 0.8434 & 0.5022 & 0.5120 & 0.3811 \\
\hline MAE & 0.0058 & 0.0129 & 0.0066 & 0.0047 & 0.0049 & 0.0040 \\
\hline RMSE & 0.0208 & 0.0413 & 0.0232 & 0.0141 & 0.0180 & 0.0156 \\
\hline Train time (s) & 0.0156 & 0.1233 & 1.5160 & 6.7440 & 0.0312 & 0.0938 \\
\hline
\end{tabular}
other models, which demonstrates that MAF-DESN has better local fitting ability in one-dimension-input task.

\begin{tabular}{|c|c|c|c|c|c|c|}
\hline & ESN & ESN-IP & ESN-PSO & LSTM & DESN & MAF-DESN \\
\hline$R$-square & 0.9742 & 0.9740 & 0.9742 & 0.9738 & 0.9742 & 0.9720 \\
\hline$R$ & 0.9870 & 0.9869 & 0.9870 & 0.9868 & 0.9870 & 0.9859 \\
\hline SSE & 5765.57 & 6065.75 & 6194.59 & 7801.07 & 6710.41 & 3928.24 \\
\hline MAE & 2.0312 & 2.1002 & 2.1145 & 2.3658 & 2.2101 & 1.7313 \\
\hline RMSE & 8.3345 & 8.2492 & 8.6391 & 9.6948 & 8.9915 & 6.8796 \\
\hline Train time (s) & 0.0012 & 0.0059 & 1.1725 & 6.0889 & 0.0026 & 0.0156 \\
\hline
\end{tabular}

Table 2. Daily foreign exchange rates (German/US).

Table 3. IBM common stock closing prices. 
Table 4. Chaotic far-infrared laser.

\begin{tabular}{|c|c|c|c|c|c|c|}
\hline & ESN & ESN-IP & ESN-PSO & LSTM & DESN & MAF-DESN \\
\hline$R$-square & 0.3799 & 0.3449 & 0.3889 & 0.3279 & 0.6487 & 0.9537 \\
\hline$R$ & 0.6164 & 0.5873 & 0.6236 & 0.5726 & 0.8054 & 0.9766 \\
\hline SSE & 368673 & 401739 & 373945 & 297059 & 164087 & 66188 \\
\hline MAE & 8.0786 & 8.4742 & 8.2035 & 7.7368 & 4.9021 & 3.4042 \\
\hline RMSE & 33.6200 & 35.1077 & 33.8669 & 30.1535 & 22.3931 & 14.3494 \\
\hline Train time (s) & 0.3700 & 0.0313 & 1.3734 & 15.2609 & 0.0156 & 0.0156 \\
\hline
\end{tabular}
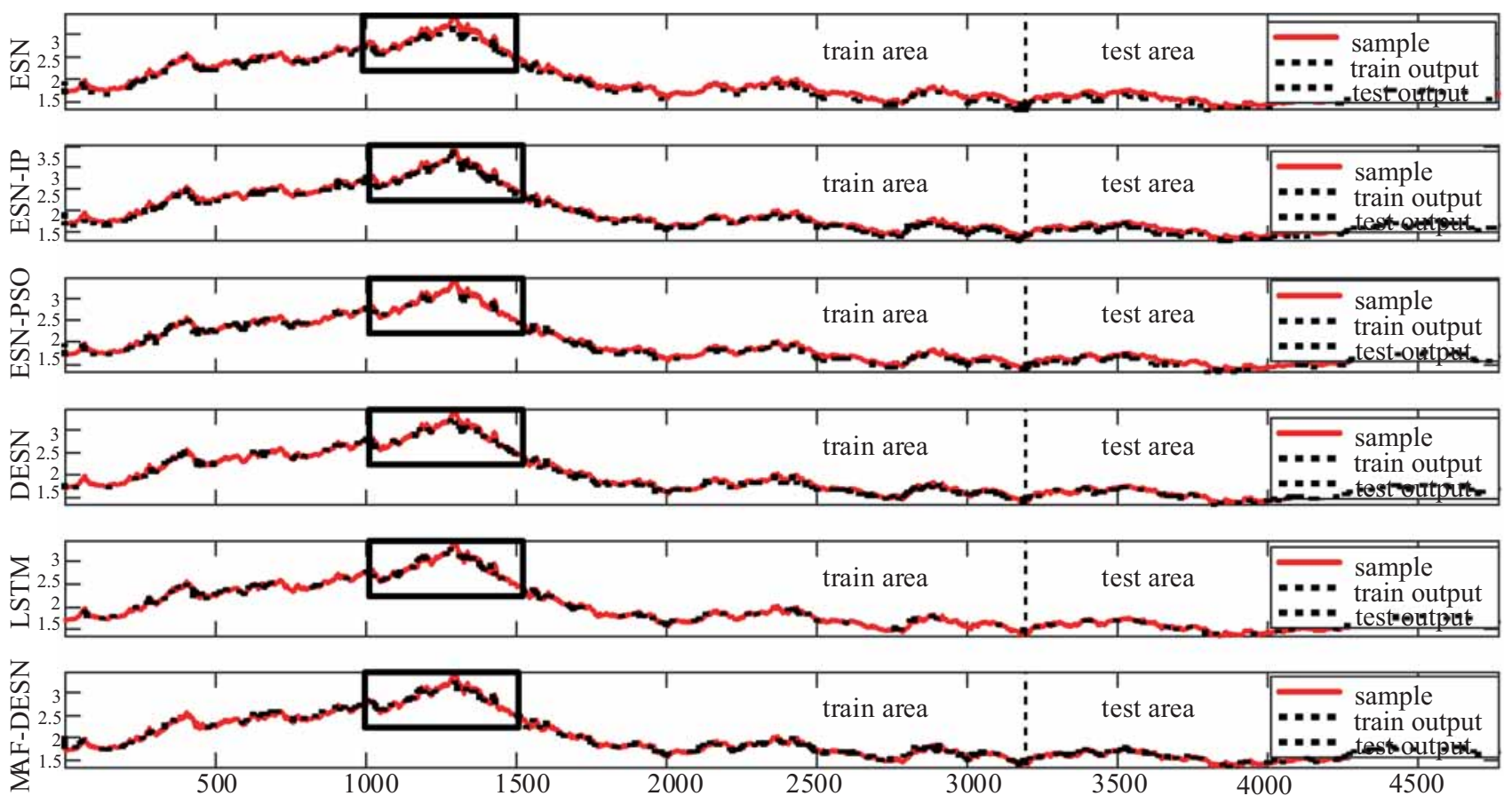

Figure 4. Daily foreign exchange rates (German/US) (target and network prediction outputs).

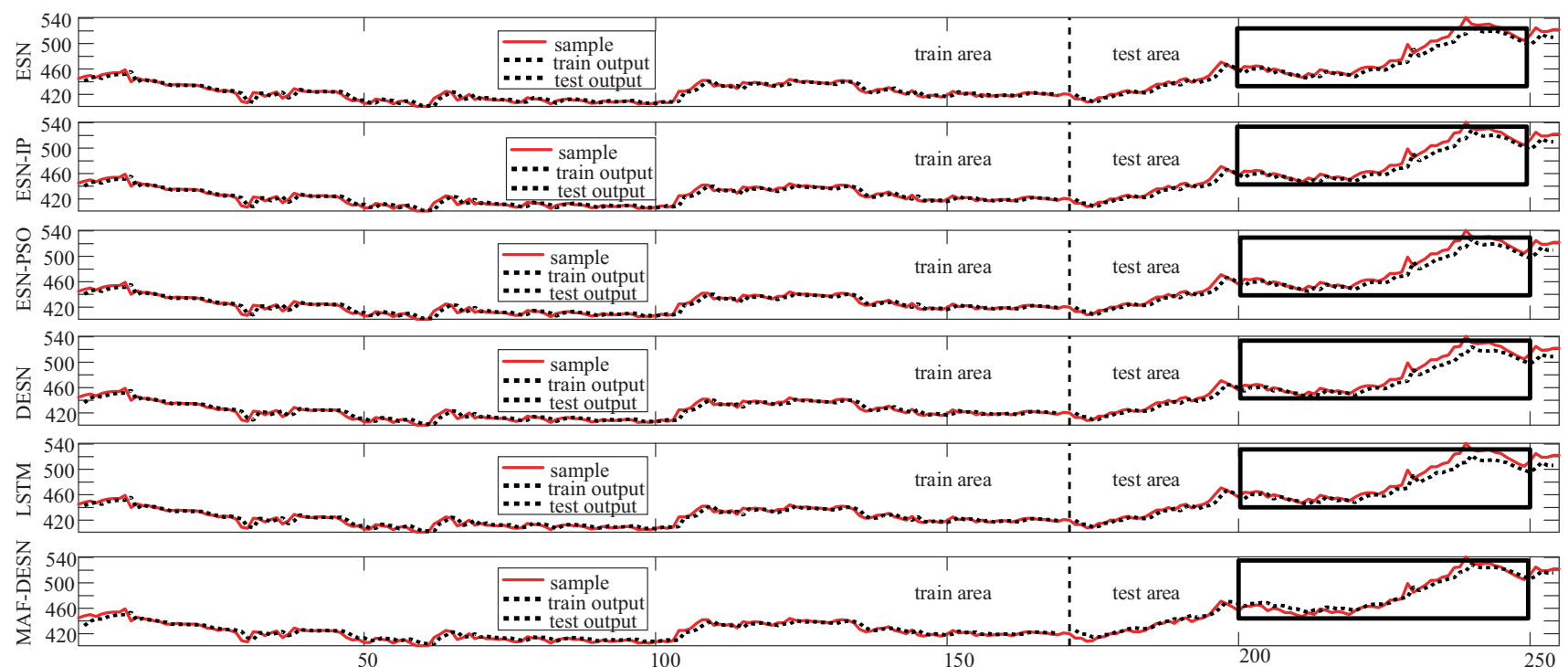

Figure 5. IBM common stock closing prices (target and network prediction outputs). 

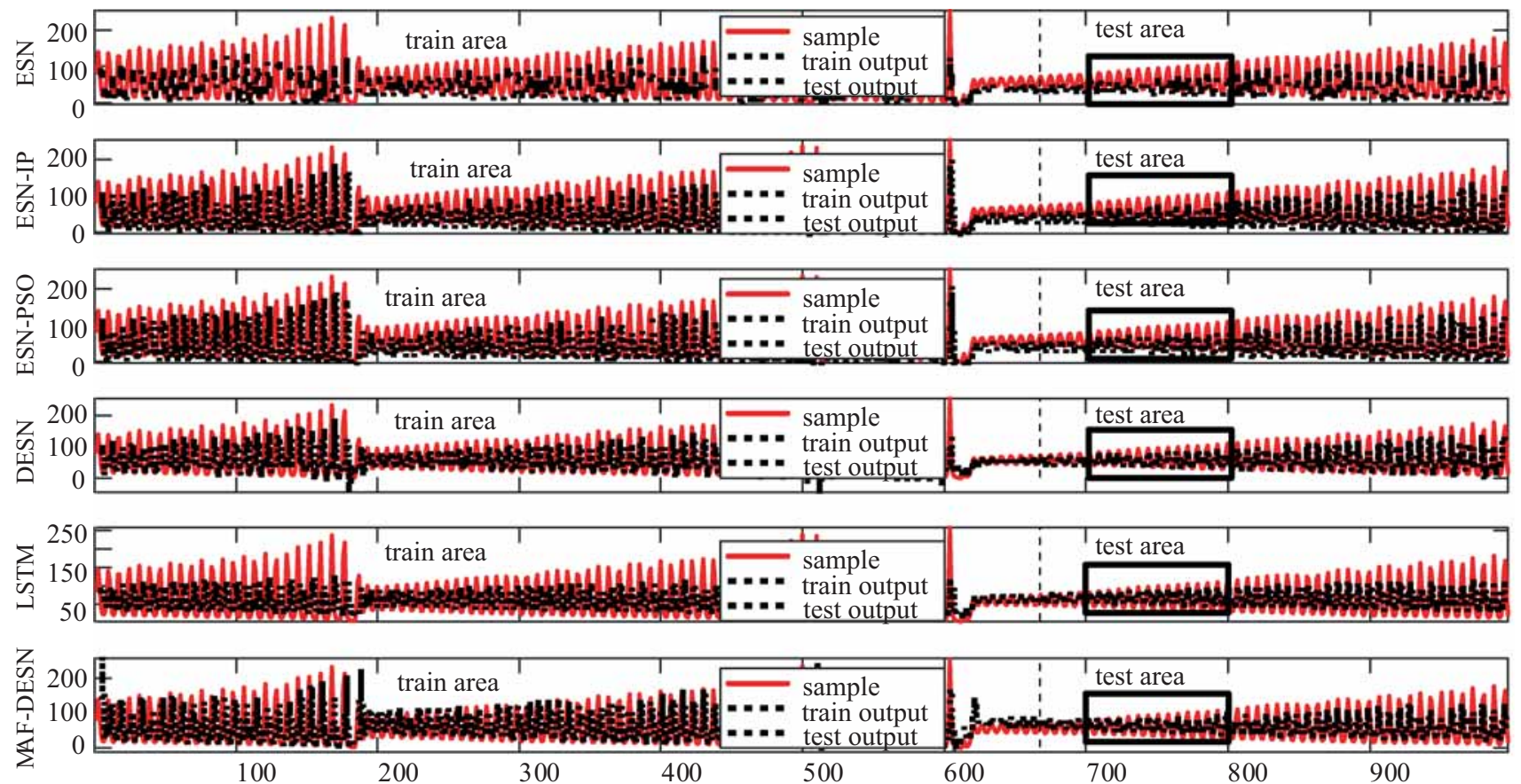

Figure 6. Chaotic far-infrared laser (target and network prediction outputs).
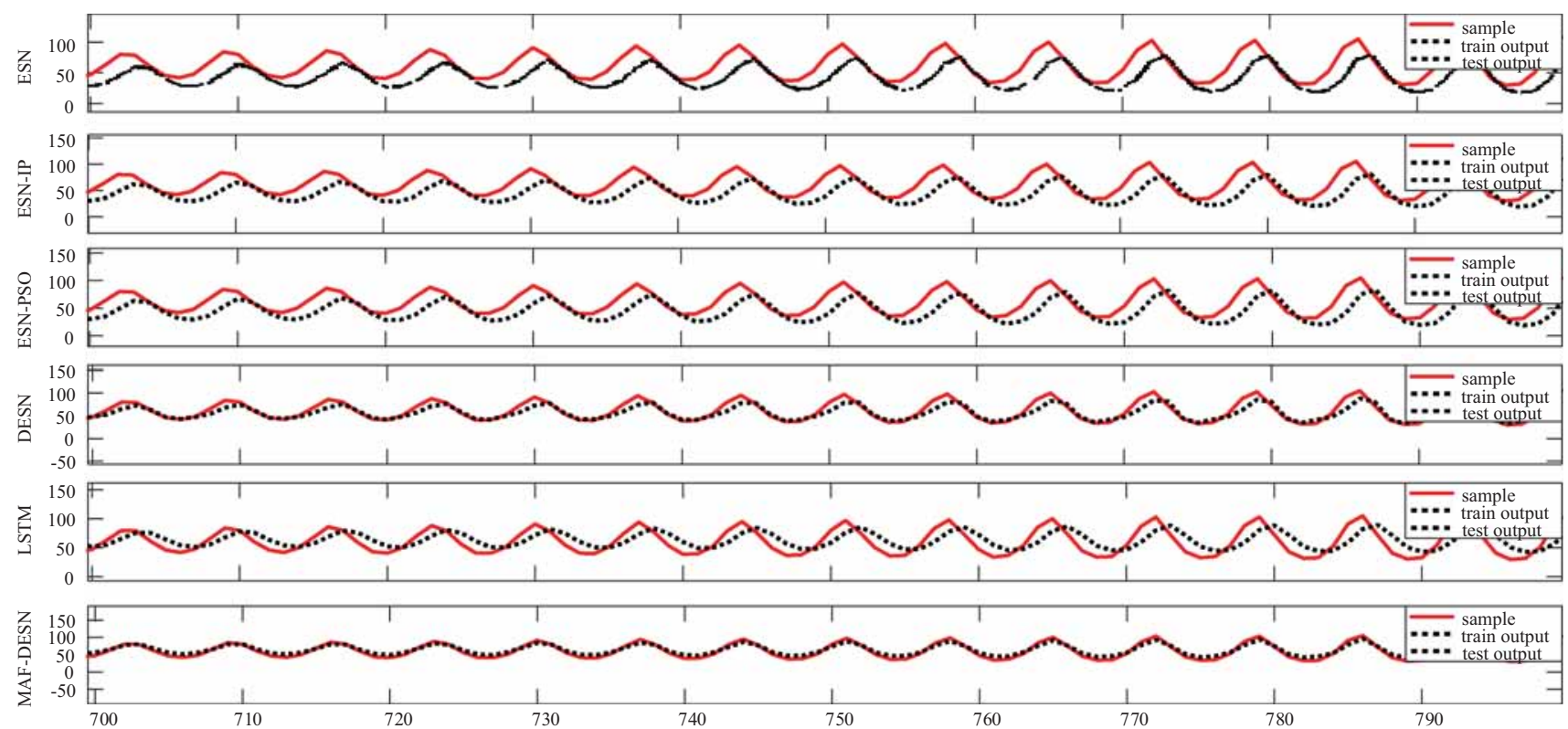

Figure 7. The local image (black frame) of figure 6 enlarged (target and network prediction outputs).

\subsection{MAF-DESN dealing with high-dimension- input task}

In this section, high-dimension-input task is downloaded from web (http://lib.stat.cmu.edu/datasets/). The input is an eight-dimensional vector consisting of temperature, relative humidity, carbon monoxide, sulphur dioxide, nitrogen dioxide, hydrocarbons, ozone and particulates, which is a quantitative digital number describing the degree of environmental pollution. The output is a threedimensional vector consisting of total mortality, respiratory mortality and cardiovascular mortality, which is disease mortality caused by environmental pollution. Since environmental variation is related to time, models with cyclic structures are used to make disease mortality prediction. 
Table 5. Pollution-caused mortality (Y1, Y2 and Y3, respectively, correspond to total mortality, respiratory mortality and cardiovascular mortality).

\begin{tabular}{|c|c|c|c|c|c|c|}
\hline & & ESN & ESN-IP & LSTM & DESN & $\begin{array}{l}\text { MAF- } \\
\text { DESN }\end{array}$ \\
\hline \multirow[t]{5}{*}{ Y1 } & $R$-square & 0.2682 & 0.2612 & 0.2557 & 0.2841 & 0.2923 \\
\hline & $R$ & 0.5179 & 0.5111 & 0.5056 & 0.5330 & 0.5406 \\
\hline & SSE & 18470 & 18286 & 25578 & 17976 & 14808 \\
\hline & MAE & 8.1169 & 8.0349 & 9.7860 & 8.1141 & 7.2830 \\
\hline & RMSE & 10.4853 & 10.4330 & 12.3391 & 10.3441 & 9.3885 \\
\hline \multirow[t]{5}{*}{$\mathrm{Y} 2$} & $R$-square & 0.1556 & 0.1510 & 0.1048 & 0.1423 & 0.1665 \\
\hline & $R$ & 0.3945 & 0.3886 & 0.3238 & 0.3773 & 0.4080 \\
\hline & SSE & 668 & 682 & 718 & 560 & 714 \\
\hline & MAE & 1.5772 & 1.5925 & 1.5862 & 1.4127 & 1.6500 \\
\hline & RMSE & 1.9935 & 2.0149 & 2.0672 & 1.8260 & 2.0609 \\
\hline \multirow[t]{5}{*}{ Y3 } & $R$-square & 0.3862 & 0.3869 & 0.3830 & 0.3975 & 0.4274 \\
\hline & $R$ & 0.6214 & 0.6220 & 0.6189 & 0.6305 & 0.6538 \\
\hline & SSE & 11850 & 11510 & 18155 & 13383 & 6663 \\
\hline & MAE & 7.1160 & 6.9702 & 8.7862 & 7.6876 & 5.2221 \\
\hline & RMSE & 8.3984 & 8.2771 & 10.3956 & 8.9254 & 6.2977 \\
\hline \multicolumn{2}{|c|}{ Train time (s) } & 0.0156 & 0.0156 & 20.4088 & 0.0313 & 0.313 \\
\hline
\end{tabular}

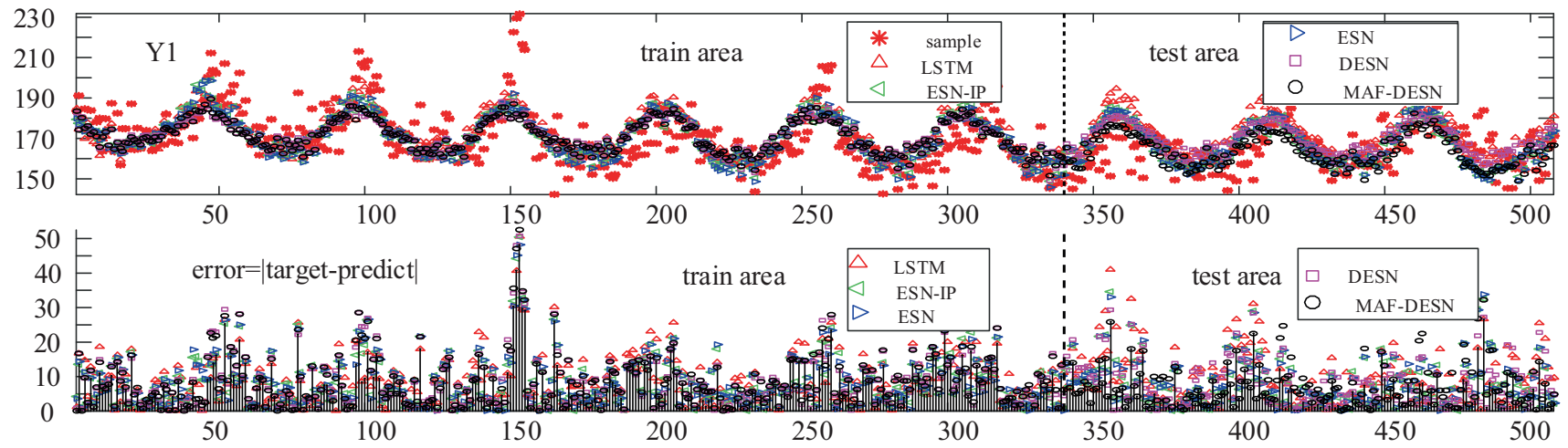

Figure 8. Total mortality.
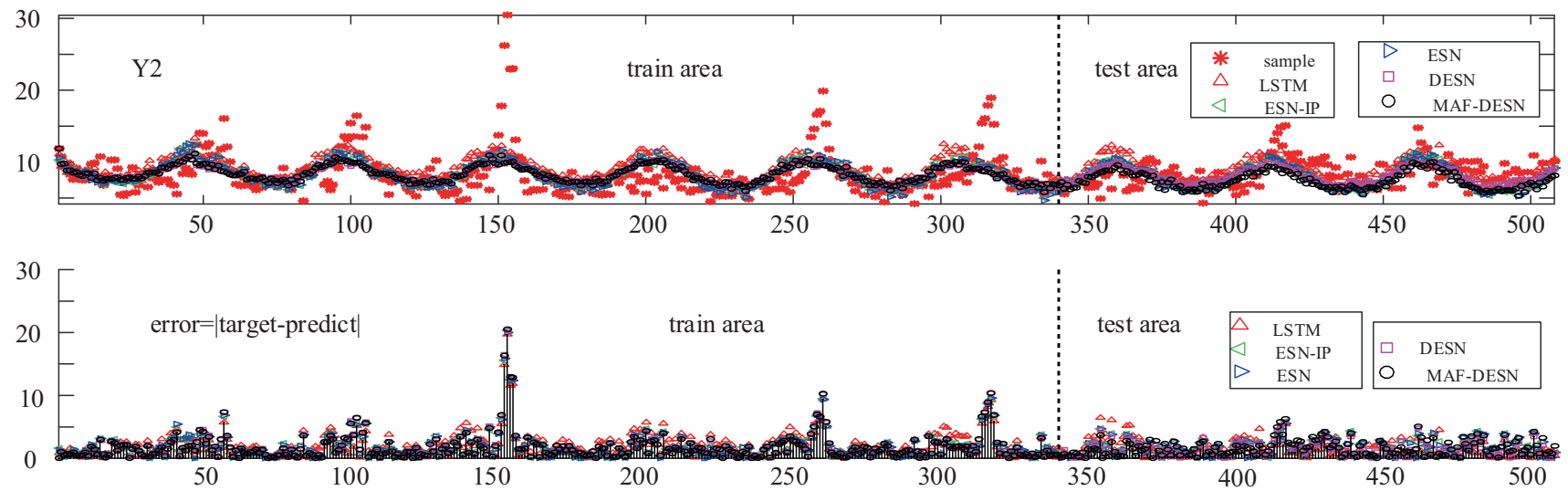

Figure 9. Respiratory mortality. 

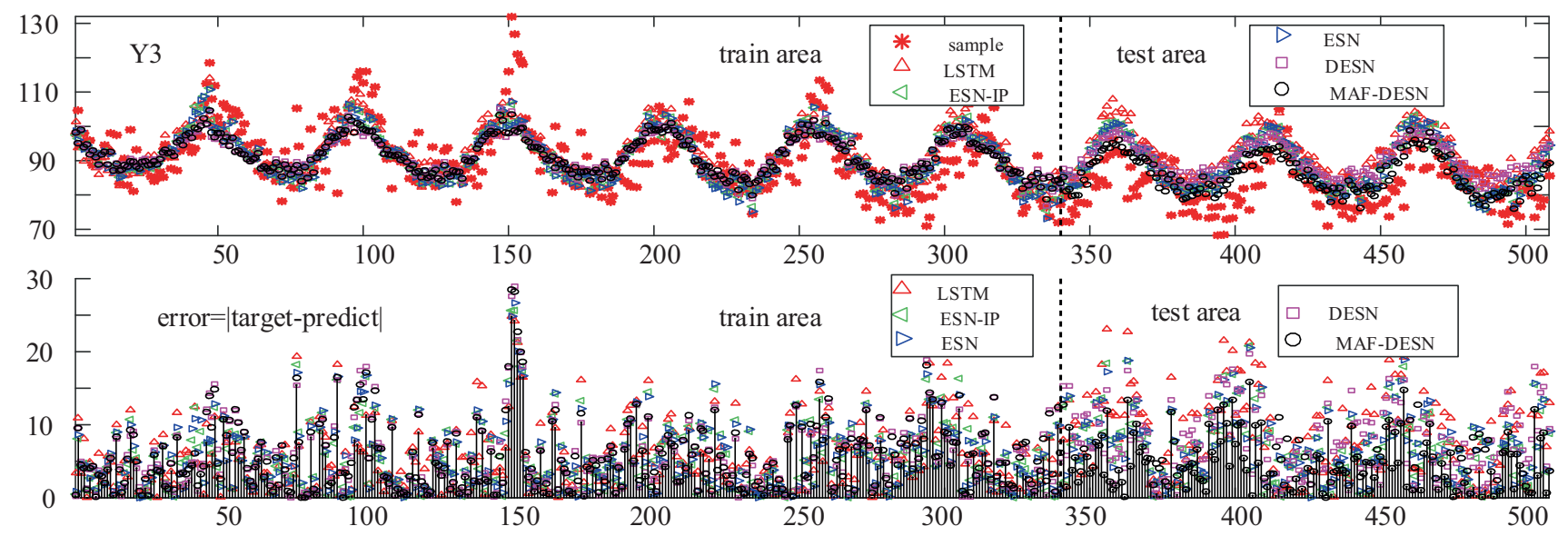

Figure 10. Cardiovascular mortality.

In this simulation, model size is 20 because the disease mortality prediction task is more complicated than the aforementioned tasks. From table 5, $R$-square and $R$ of MAF-DESN in total mortality, respiratory mortality and cardiovascular mortality prediction are slightly closer to 1 than those of other models, and SSE, MAE and RMSE of MAF-DESN model are closer to 0 than those of other models, which demonstrate that MAF-DESN in high-dimension-input task has advantage over other models considered here. Figures 8-10 show the comparison details of prediction mortality

\section{Conclusions}

In this paper, we introduced a novel model named MAFDESN, which uses two or more activation functions to substitute the original reservoir activation function in one layer, and the scaling factor of activation functions varied layer by layer. The simulation shows that MAF-DESN possesses stronger explanatory power and better fitting ability with little increase of time consumption. However, in our simulations, the parameters are tuned by experience, which may not be the best fitting parameters; besides, the ill-posed problem of DESN is not solved in the proposed model. In the future, we attempt to apply the steepest descent method to find the best fitting parameters, as well as apply some dimensionality reduction algorithm (such as Laplacian eigenmap [4], Principal Component Analysis, singular value decomposition, Empirical Mode Decomposition, etc.) to refine the ill-posed problem

\section{Acknowledgements}

This work is supported by Sichuan Science and Technology Program (2019YFSY0016). The authors declare that there is no conflict of interest regarding the publication of this paper.

\section{Appendix A}

Before proving that $\left\|\boldsymbol{x}(n+1)-\boldsymbol{x}^{\prime}(n+1)\right\| \leq \| \boldsymbol{x}(n)-$ $x^{\prime}(n) \|$, we describe the relationship between $\left\|\boldsymbol{x}(n+1)-\boldsymbol{x}^{\prime}(n+1)\right\|$ and $\left\|\boldsymbol{x}^{(l)}(n+1)-\boldsymbol{x}^{\prime(l)}(n+1)\right\|$.

"(a)": According to aforementioned gathering of reservoir states $\boldsymbol{x}(n)$ and reservoir states of $l$ th layer $\boldsymbol{x}^{(l)}(n)$, we unfold $\boldsymbol{x}(n)$ as the following vector:

$$
\begin{aligned}
& \boldsymbol{x}(n)=
\end{aligned}
$$

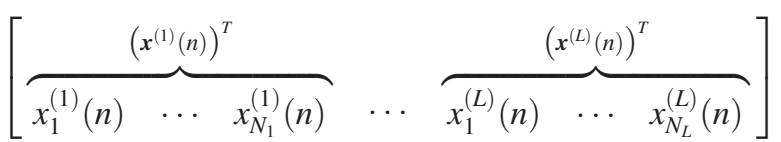

where superscript denotes reservoir location, the maximum number of layers is $L$, subscript denotes neuron location of current reservoir and maximum number of reservoir neurons is $N_{l}(l=1, \cdots L)$. Then, substituting Eq. (A.1) into $\left\|\boldsymbol{x}(n+1)-\boldsymbol{x}^{\prime}(n+1)\right\|$, we obtain following equations:

$$
\begin{aligned}
& \left\|\boldsymbol{x}(n+1)-\boldsymbol{x}^{\prime}(n+1)\right\| \\
& \quad\left\|\begin{array}{c}
x_{1}^{(1)}(n+1)-x_{1}^{\prime(1)}(n+1) \\
\vdots \\
x_{N_{1}}^{(1)}(n+1)-x_{N_{1}}^{\prime(1)}(n+1) \\
\vdots \\
x_{1}^{(L)}(n+1)-x_{1}^{\prime(L)}(n+1) \\
\vdots \\
x_{N_{L}}^{(L)}(n+1)-x_{N_{L}}^{\prime(L)}(n+1)
\end{array}\right\| \\
& =\sqrt{\sum_{l=1}^{L} \sum_{j=1}^{N_{l}}\left(x_{j}^{(l)}(n+1)-x_{j}^{\prime(l)}(n+1)\right)^{2}},
\end{aligned}
$$




$$
\begin{aligned}
\left\|\boldsymbol{x}^{(l)}(n+1)-\boldsymbol{x}^{\prime(l)}(n+1)\right\| \\
=\left\|\begin{array}{c}
x_{1}^{(l)}(n+1)-x_{1}^{\prime(l)}(n+1) \\
\vdots \\
x_{N_{l}}^{(l)}(n+1)-x_{N_{l}}^{(l)}(n+1)
\end{array}\right\| \\
=\sqrt{\sum_{j=1}^{N_{l}}\left(x_{j}^{(l)}(n+1)-x_{j}^{(l)}(n+1)\right)^{2}} .
\end{aligned}
$$

According to Eqs. (A.2) and (A.3), we obtain the relationship between $\left\|\boldsymbol{x}(n+1)-\boldsymbol{x}^{\prime}(n+1)\right\|$ and $\left\|\boldsymbol{x}^{(l)}(n+1)-\boldsymbol{x}^{(l)}(n+1)\right\|$ as follows:

$$
\begin{aligned}
\| \boldsymbol{x}(n+1) & -\boldsymbol{x}^{\prime}(n+1) \|^{2} \\
& =\sum_{l=1}^{L}\left\|\boldsymbol{x}^{(l)}(n+1)-\boldsymbol{x}^{\prime(l)}(n+1)\right\|^{2} .
\end{aligned}
$$

"(b)": Now, the proof of Inequation (9) is described here. According to Eq. (A.4), proof of $\left\|\boldsymbol{x}(n+1)-\boldsymbol{x}^{\prime}(n+1)\right\| \leq\left\|\boldsymbol{x}(n)-\boldsymbol{x}^{\prime}(n)\right\|$ is equivalent to that of

$$
\begin{aligned}
& \sum_{l=1}^{L}\left\|\boldsymbol{x}^{(l)}(n+1)-\boldsymbol{x}^{(l)}(n+1)\right\|^{2} \\
& \quad \leq \sum_{l=1}^{L}\left\|\boldsymbol{x}^{(l)}(n)-\boldsymbol{x}^{(l)}(n)\right\|^{2} .
\end{aligned}
$$

Hence, we prove the latter inequation as follows:

$$
\begin{aligned}
\sum_{l=1}^{L} & \left\|\boldsymbol{x}^{(l)}(n+1)-\boldsymbol{x}^{(l)}(n+1)\right\|^{2} \\
= & \sum_{l=1}^{L} \|\left(1-a^{(l)}\right)\left(\boldsymbol{x}^{(l)}(n)-\boldsymbol{x}^{\prime(l)}(n)\right) \\
& +a^{(l)}\left(f^{(l)}\left(\boldsymbol{x}_{i n}^{(l)}(n+1)\right)-f^{(l)}\left(\boldsymbol{x}_{\boldsymbol{i n}}^{(l)}(n+1)\right)\right) \|^{2} \\
\leq & \sum_{l=1}^{L} \|\left(1-a^{(l)}\right)\left(\boldsymbol{x}^{(l)}(n)-\boldsymbol{x}^{\prime(l)}(n)\right) \\
& +a^{(l)}\left(\boldsymbol{x}_{i n}^{(l)}(n+1)-\boldsymbol{x}_{i n}^{(l)}(n+1)\right) \|^{2} \\
= & \sum_{l=1}^{L} \|\left(\left(1-a^{(l)}\right) \boldsymbol{E}^{(l)}+a^{(l)} W^{(l)}\right)\left(\boldsymbol{x}^{(l)}(n)-\boldsymbol{x}^{(l)}(n)\right) \\
& +a^{(l)} \boldsymbol{W}_{i n}^{(l)}\left(\boldsymbol{i}^{(l)}(n+1)-\boldsymbol{i}^{(l)}(n+1)\right) \|^{2} \\
\leq & \sum_{l=1}^{L}\left(\left\|\left(\left(1-a^{(l)}\right) \boldsymbol{E}^{(l)}+a^{(l)} \boldsymbol{W}^{(l)}\right)\left(\boldsymbol{x}^{(l)}(n)-\boldsymbol{x}^{(l)}(n)\right)\right\|\right. \\
& \left.+\left\|a^{(l)} \boldsymbol{W}_{i n}^{(l)}\left(\boldsymbol{i}^{(l)}(n+1)-\boldsymbol{i}^{(l)}(n+1)\right)\right\|\right)^{2} \\
\leq & \sum_{l=1}^{L}\left\|\left(\left(1-a^{(l)}\right) \boldsymbol{E}^{(l)}+a^{(l)} \boldsymbol{W}^{(l)}\right)\left(\boldsymbol{x}^{(l)}(n)-\boldsymbol{x}^{\prime(l)}(n)\right)\right\|^{2} \\
& +\sum_{l=1}^{L}\left\|a^{(l)} \boldsymbol{W}_{i n}^{(l)}\left(\boldsymbol{i}^{(l)}(n+1)-\boldsymbol{i}^{\prime(l)}(n+1)\right)\right\|^{2} .
\end{aligned}
$$

According to Eq. (2), we know that

$$
\left\{\begin{array}{l}
\boldsymbol{i}^{(l)}(n+1)-\boldsymbol{i}^{(l)}(n+1)=0, l=1 \\
\boldsymbol{i}^{(l)}(n+1)-\boldsymbol{i}^{(l)}(n+1)=\boldsymbol{x}^{(l-1)}(n+1)-\boldsymbol{x}^{(l-1)}(n+1), l>1
\end{array}\right.
$$

Then

$$
\begin{aligned}
& \left\|\boldsymbol{x}(n+1)-\boldsymbol{x}^{\prime}(n+1)\right\|^{2}=\sum_{l=1}^{L}\left\|\boldsymbol{x}^{(l)}(n+1)-\boldsymbol{x}^{\prime(l)}(n+1)\right\|^{2} \\
& \leq \sum_{l=1}^{L}\left\|\left(\left(1-a^{(l)}\right) \boldsymbol{E}^{(l)}+a^{(l)} \boldsymbol{W}^{(l)}\right)\left(\boldsymbol{x}^{(l)}(n)-\boldsymbol{x}^{(l)}(n)\right)\right\|^{2} \\
& +\sum_{l=2}^{L}\left\|a^{(l)} \boldsymbol{W}_{i n}^{(l)}\left(\boldsymbol{x}^{(l-1)}(n+1)-\boldsymbol{x}^{(l-1)}(n+1)\right)\right\|^{2} \\
& \leq \sum_{l=1}^{L}\left\|\left(\left(1-a^{(l)}\right) \boldsymbol{E}^{(l)}+a^{(l)} \boldsymbol{W}^{(l)}\right)\left(\boldsymbol{x}^{(l)}(n)-\boldsymbol{x}^{(l)}(n)\right)\right\|^{2} \\
& +\sum_{l=1}^{L-1}\left\|a^{(l+1)} \boldsymbol{W}_{\boldsymbol{i n}}^{(l+1)}\left(\boldsymbol{x}^{(l)}(n+1)-\boldsymbol{x}^{(l)}(n+1)\right)\right\|^{2} \\
& \leq \sum_{l=1}^{L}\left(1-a^{(l)}+a^{(l)} \Lambda^{(l)}\right)^{2}\left\|\left(\boldsymbol{x}^{(l)}(n)-\boldsymbol{x}^{(l)}(n)\right)\right\|^{2} \\
& +\sum_{l=1}^{L-1}\left(a^{(l+1)} \Lambda_{\mathrm{in}}^{(l+1)}\right)^{2}\left\|\left(\boldsymbol{x}^{(l)}(n+1)-\boldsymbol{x}^{(l)}(n+1)\right)\right\|^{2} \\
& \Rightarrow \sum_{l=1}^{L}\left\|\boldsymbol{x}^{(l)}(n+1)-\boldsymbol{x}^{(l)}(n+1)\right\|^{2} \\
& -\sum_{l=1}^{L-1}\left(a^{(l+1)} \Lambda_{\mathrm{in}}^{(l+1)}\right)^{2}\left\|\left(\boldsymbol{x}^{(l)}(n+1)-\boldsymbol{x}^{\prime(l)}(n+1)\right)\right\|^{2} \\
& \leq \sum_{l=1}^{L}\left(1-a^{(l)}+a^{(l)} \Lambda^{(l)}\right)^{2}\left\|\left(\boldsymbol{x}^{(l)}(n)-\boldsymbol{x}^{(l)}(n)\right)\right\|^{2} \\
& \Rightarrow \sum_{l=1}^{L-1}\left(1-\left(a^{(l+1)} \Lambda_{\mathrm{in}}^{(l+1)}\right)^{2}\right)\left\|\boldsymbol{x}^{(l)}(n+1)-\boldsymbol{x}^{\prime(l)}(n+1)\right\|^{2} \\
& +\left\|\boldsymbol{x}^{(L)}(n+1)-\boldsymbol{x}^{(L)}(n+1)\right\|^{2} \\
& \leq \sum_{l=1}^{L}\left(1-a^{(l)}+a^{(l)} \Lambda^{(l)}\right)^{2}\left\|\left(\boldsymbol{x}^{(l)}(n)-\boldsymbol{x}^{(l)}(n)\right)\right\|^{2} \text {. }
\end{aligned}
$$

Unfolding of Inequation (A.8) is as follows: when $l=1,2, \cdots L-1$

$$
\begin{aligned}
\left\|\boldsymbol{x}^{(l)}(n+1)-\boldsymbol{x}^{(l)}(n+1)\right\|^{2} & \\
& \leq \frac{\left(1-a^{(l)}+a^{(l)} \Lambda^{(l)}\right)^{2}}{1-\left(a^{(l+1)} \Lambda_{\mathrm{in}}^{(l+1)}\right)^{2}}\left\|\boldsymbol{x}^{(l)}(n)-\boldsymbol{x}^{(l)}(n)\right\|^{2} .
\end{aligned}
$$

When $l=L$

$$
\begin{aligned}
& \left\|\boldsymbol{x}^{(l)}(n+1)-\boldsymbol{x}^{(l)}(n+1)\right\|^{2} \\
& \quad \leq\left(1-a^{(l)}+a^{(l)} \Lambda^{(l)}\right)^{2}\left\|\boldsymbol{x}^{(l)}(n)-\boldsymbol{x}^{(l)}(n)\right\|^{2} .
\end{aligned}
$$

According to Inequations (A.9) and (A.10), when following Inequations (A.11) and (A.12)

$$
\begin{aligned}
& \frac{\left(1-a^{(l)}+a^{(l)} \Lambda^{(l)}\right)^{2}}{1-\left(a^{(l+1)} \Lambda_{\mathrm{in}}^{(l+1)}\right)^{2}}\left\|\boldsymbol{x}^{(l)}(n)-\boldsymbol{x}^{(l)}(n)\right\|^{2} \\
& \quad \leq\left\|\boldsymbol{x}^{(L)}(n)-\boldsymbol{x}^{(L)}(n)\right\|^{2}, l=1,2, \cdots L-1
\end{aligned}
$$

$$
\begin{aligned}
(1- & \left.a^{(l)}+a^{(l)} \Lambda^{(l)}\right)^{2}\left\|\boldsymbol{x}^{(l)}(n)-\boldsymbol{x}^{\prime(l)}(n)\right\|^{2} \\
& \leq\left\|\boldsymbol{x}^{(L)}(n+1)-\boldsymbol{x}^{(L)}(n+1)\right\|^{2}, l=L
\end{aligned}
$$

are satisfied, then 


$$
\left\|\boldsymbol{x}^{(l)}(n+1)-\boldsymbol{x}^{(l)}(n+1)\right\|^{2} \leq\left\|\boldsymbol{x}^{(l)}(n)-\boldsymbol{x}^{(l)}(n)\right\|^{2}
$$

are satisfied, which means that

$\sum_{l=1}^{L}\left\|\boldsymbol{x}^{(l)}(n+1)-\boldsymbol{x}^{\prime(l)}(n+1)\right\|^{2} \leq \sum_{l=1}^{L}\left\|\boldsymbol{x}^{(l)}(n)-\boldsymbol{x}^{\prime(l)}(n)\right\|^{2}$

are satisfied, which also means that $\left\|\boldsymbol{x}(n+1)-\boldsymbol{x}^{\prime}(n+1)\right\|^{2} \leq\left\|\boldsymbol{x}(n)-\boldsymbol{x}^{\prime}(n)\right\|^{2}$ are satisfied. We transform Inequations (A.11) and (A.12) into the following form:

$$
\left\{\begin{array}{l}
\frac{\left(1-a^{(l)}+a^{(l)} \Lambda^{(l)}\right)^{2}}{1-\left(a^{(l+1)} \Lambda_{\mathrm{in}}^{(l+1)}\right)^{2}} \leq 1, l=1,2, \cdots L-1 \\
\left(1-a^{(l)}+a^{(l)} \Lambda^{(l)}\right)^{2} \leq 1, l=L
\end{array}\right.
$$

Then, the proof process is completed.

\section{Appendix B}

According to Eq. (A.4), proof of Inequation (13) is equivalent to that of (A.14). Hence, we prove the latter Inequation (A.14) as follows:

$$
\begin{aligned}
\sum_{l=1}^{L} & \left\|\boldsymbol{x}^{(l)}(n+1)-\boldsymbol{x}^{\prime(l)}(n+1)\right\|^{2} \\
= & \sum_{l=1}^{L} \|\left(1-a^{(l)}\right)\left(\boldsymbol{x}^{(l)}(n)-\boldsymbol{x}^{\prime(l)}(n)\right) \\
& +\sum_{i=1}^{N_{l}^{l}}\left(\lambda_{i}^{(l)}\left(f_{i}^{(l)}\left(\boldsymbol{x}_{i n}^{(l)}(n+1)\right)-f_{i}^{(l)}\left(\boldsymbol{x}_{i n}^{\prime(l)}(n+1)\right)\right)\right) \|^{2} \\
\leq & \sum_{l=1}^{L} \|\left(1-a^{(l)}\right)\left(\boldsymbol{x}^{(l)}(n)-\boldsymbol{x}^{\prime(l)}(n)\right) \\
& +\sum_{i=1}^{N_{l}^{l}}\left(\lambda_{i}^{(l)}\left(\boldsymbol{x}_{i n}^{(l)}(n+1)-\boldsymbol{x}_{i n}^{(l)}(n+1)\right)\right) \|^{2} \\
\leq & \sum_{l=1}^{L} \|\left(\left(1-a^{(l)}\right) \boldsymbol{E}^{(l)}+\sum_{i=1}^{N_{l}^{l}} \lambda_{i}^{(l)} \boldsymbol{W}^{(l)}\right)\left(\boldsymbol{x}^{(l)}(n)-\boldsymbol{x}^{(l)}(n)\right) \\
& +\sum_{i=1}^{N_{l}^{l}}\left(\lambda_{i}^{(l)} \boldsymbol{W}_{i n}^{(l)}\left(\boldsymbol{i}^{(l)}(n+1)-\boldsymbol{i}^{\prime(l)}(n+1)\right)\right) \|^{2} \\
\leq & \sum_{l=1}^{L}\left\|\left(\left(1-a^{(l)}\right) \boldsymbol{E}^{(l)}+\sum_{i=1}^{N_{l}^{l}} \lambda_{i}^{(l)} \boldsymbol{W}^{(l)}\right)\left(\boldsymbol{x}^{(l)}(n)-\boldsymbol{x}^{(l)}(n)\right)\right\|^{2} \\
& +\sum_{l=1}^{L}\left\|\sum_{i=1}^{N_{l}^{l}}\left(\lambda_{i}^{(l)} \boldsymbol{W}_{i n}^{(l)}\left(\boldsymbol{i}^{(l)}(n+1)-\boldsymbol{i}^{(l)}(n+1)\right)\right)\right\|^{2} \\
\leq & \sum_{l=1}^{L}\left(1-a^{(l)}+\sum_{i=1}^{N_{l}^{l}}\left|\lambda_{i}^{(l)}\right| \Lambda^{(l)}\right)^{2}\left\|\boldsymbol{x}^{(l)}(n)-\boldsymbol{x}^{\prime(l)}(n)\right\|^{2} \\
& +\sum_{l=1}^{L}\left(\sum_{i=1}^{N_{i}^{l}}\left|\lambda_{i}^{(l)}\right| \Lambda_{\mathrm{in}}^{(l)}\right)^{2}\left\|\boldsymbol{i}^{(l)}(n+1)-\boldsymbol{i}^{\prime(l)}(n+1)\right\|^{2} .
\end{aligned}
$$

According to Eq. (A.7)

$$
\begin{aligned}
\left\|\boldsymbol{x}(n+1)-\boldsymbol{x}^{\prime}(n+1)\right\|^{2} \\
=\sum_{l=1}^{L}\left\|\boldsymbol{x}^{(l)}(n+1)-\boldsymbol{x}^{\prime(l)}(n+1)\right\|^{2} \\
\leq \sum_{l=1}^{L}\left(1-a^{(l)}+\sum_{i=1}^{N_{i}^{l}}\left|\lambda_{i}^{(l)}\right| \Lambda^{(l)}\right)^{2}\left\|\boldsymbol{x}^{(l)}(n)-\boldsymbol{x}^{\prime(l)}(n)\right\|^{2} \\
\quad+\sum_{l=2}^{L}\left(\sum_{i=1}^{N_{i}^{l}}\left|\lambda_{i}^{(l)}\right| \Lambda_{\mathrm{in}}^{(l)}\right)^{2}\left\|\boldsymbol{x}^{(l-1)}(n+1)-\boldsymbol{x}^{(l-1)}(n+1)\right\|^{2} \\
\leq \sum_{l=1}^{L}\left(1-a^{(l)}+\sum_{i=1}^{N_{l}^{l}}\left|\lambda_{i}^{(l)}\right| \Lambda^{(l)}\right)^{2}\left\|\boldsymbol{x}^{(l)}(n)-\boldsymbol{x}^{\prime(l)}(n)\right\|^{2} \\
\quad+\sum_{l=1}^{L-1}\left(\sum_{i=1}^{N_{i}^{l+1}}\left|\lambda_{i}^{(l+1)}\right| \Lambda_{\mathrm{in}}^{(l+1)}\right)^{2}\left\|\boldsymbol{x}^{(l)}(n+1)-\boldsymbol{x}^{(l)}(n+1)\right\|^{2}
\end{aligned}
$$

Transform Inequation (B.2) as follows:

$$
\begin{aligned}
\sum_{l=1}^{L} & \left\|\boldsymbol{x}^{(l)}(n+1)-\boldsymbol{x}^{\prime(l)}(n+1)\right\|^{2} \\
& -\sum_{l=1}^{L-1}\left(\sum_{i=1}^{N_{l}^{l+1}}\left|\lambda_{i}^{(l+1)}\right| \Lambda_{\mathrm{in}}^{(l+1)}\right)^{2}\left\|\left(\boldsymbol{x}^{(l)}(n+1)-\boldsymbol{x}^{(l)}(n+1)\right)\right\|^{2} \\
& \leq \sum_{l=1}^{L}\left(1-a^{(l)}+\sum_{i=1}^{N_{l}^{l}}\left|\lambda_{i}^{(l)}\right| \Lambda^{(l)}\right)^{2}\left\|\boldsymbol{x}^{(l)}(n)-\boldsymbol{x}^{(l)}(n)\right\|^{2}
\end{aligned}
$$

Transform Inequation (B.3) as follows:

$$
\begin{aligned}
& \sum_{l=1}^{L-1}\left(1-\left(\sum_{i=1}^{N_{\lambda}^{l+1}}\left|\lambda_{i}^{(l+1)}\right| \Lambda_{\mathrm{in}}^{(l+1)}\right)^{2}\right) \\
& \left\|\boldsymbol{x}^{(l)}(n+1)-\boldsymbol{x}^{(l)}(n+1)\right\|^{2} \\
& \quad+\left\|\boldsymbol{x}^{(L)}(n+1)-\boldsymbol{x}^{\prime(L)}(n+1)\right\|^{2} \\
& \leq \sum_{l=1}^{L}\left(1-a^{(l)}+\sum_{i=1}^{N_{\lambda}^{l}}\left|\lambda_{i}^{(l)}\right| \Lambda^{(l)}\right)^{2} \\
& \left\|\left(\boldsymbol{x}^{(l)}(n)-\boldsymbol{x}^{\prime(l)}(n)\right)\right\|^{2}
\end{aligned}
$$

Similar to Appendix A, when the following inequations

$$
\begin{gathered}
\frac{\left(1-a^{(l)}+\sum_{i=1}^{N_{\lambda}^{l}}\left|\lambda_{i}^{(l)}\right| \Lambda^{(l)}\right)^{2}}{1-\left(\sum_{i=1}^{N_{\lambda}^{l+1}}\left|\lambda_{i}^{(l+1)}\right| \Lambda_{\mathrm{in}}^{(l+1)}\right)^{2}}\left\|\boldsymbol{x}^{(l)}(n)-\boldsymbol{x}^{\prime(l)}(n)\right\|^{2} \\
\leq\left\|\boldsymbol{x}^{(L)}(n)-\boldsymbol{x}^{\prime(L)}(n)\right\|^{2}, l=1,2, \cdots L-1 \\
\left(1-a^{(l)}+\sum_{i=1}^{N_{\lambda}^{l}}\left|\lambda_{i}^{(l)}\right| \Lambda^{(l)}\right)^{2}\left\|\boldsymbol{x}^{(l)}(n)-\boldsymbol{x}^{\prime(l)}(n)\right\|^{2} \\
\leq\left\|\boldsymbol{x}^{(L)}(n+1)-\boldsymbol{x}^{\prime(L)}(n+1)\right\|^{2}, l=L
\end{gathered}
$$

are satisfied, the sufficient condition is obtained. According to Inequations (B.3)-(B.6), one can obtain following inequations: 


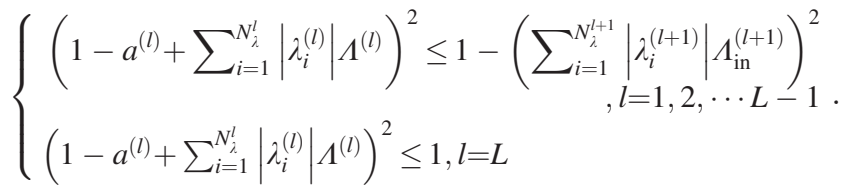

Inequation (B.7) develops to the following:

$\left\{\begin{array}{l}0<\Lambda^{(l)}<1, l=1,2, \cdots L-1 \\ 0<\Lambda^{(l)}<a^{(l)} / \sum_{i=1}^{N_{l}^{l}}\left|\lambda_{i}^{(l)}\right| \Lambda^{(l)}, l=L \\ \Lambda_{\mathrm{in}}^{(l+1)} \leq \sqrt{1-\left(1-a^{(l)}+\sum_{i=1}^{N_{\lambda}^{l}}\left|\lambda_{i}^{(l)}\right| \Lambda^{(l)}\right)^{2}} / a^{(l+1)}, l=1,2, \cdots L-1\end{array}\right.$.

Then, the proof process is completed.

\section{References}

[1] Schrauwen B et al 2008 Improving reservoirs using intrinsic plasticity. Neurocomputing 71: 1159-1171

[2] Guo Y et al 2017 Robust echo state networks based on correntropy induced loss function. Neurocomputing 267: 295-303

[3] Chen L, Qu H and Zhao J 2016 Generalized correntropy induced loss function for deep learning. In: Proceedings of the International Joint Conference on Neural Networks, IEEE, pp. 1428-1433

[4] Han M and Xu M 2018 Laplacian echo state network for multivariate time series prediction. IEEE Trans. Neural Netw. Learn. Syst. 29(1): 238-244

[5] Basterrech S and Rubino G 2017 Echo state queueing networks: a combination of reservoir computing and random neural networks. Probab. Eng. Inf. Sci. 31(4): 1-20

[6] Dorado-Moreno M et al 2016 Multiclass prediction of wind power ramp events combining reservoir computing and support vector machines. Adv. Artif. Intell. 1: 300-309

[7] Sun X et al 2017 Deep belief echo-state network and its application to time series prediction. Knowl. Based Syst. 130: 17-29

[8] Evermann J, Rehse J R and Fettke P 2017 Predicting process behaviour using deep learning. Decis. Support Syst. 100: $129-140$

[9] Wang H and Yan X 2015 Optimizing the echo state network with a binary particle swarm optimization algorithm. Knowl. Based Syst. 86: 182-193

[10] Chan P K et al 2017 Sensitivity based robust learning for stacked autoencoder against evasion attack. Neurocomputing 267: $572-580$

[11] Yang C et al 2018 Dynamical regularized echo state network for time series prediction. Neural Comput. Appl. 1-14, https://doi.org/10.1007/s00521-018-3488-z

[12] Qiao J, Wang L and Yang C 2018 Adaptive lasso echo state network based on modified Bayesian information criterion for nonlinear system modeling. Neural Comput. Appl. 1-15, https://doi.org/10.1007/s00521-018-3420-6

[13] Huang B et al 2018 Recursive Bayesian echo state network with an adaptive inflation factor for temperature prediction. Neural Comput. Appl. 29(12): 1-9

[14] Yang C et al 2018 Design of polynomial echo state networks for time series prediction. Neurocomputing 290: 148-160

[15] Malik Z K, Hussain A and Wu Q J 2017 Multilayered echo state machine: a novel architecture and algorithm. IEEE Trans. Cybern. 47(4): 1-14

[16] Gallicchio C, Micheli A and Pedrelli L 2017 Deep reservoir computing: a critical experimental analysis. Neurocomputing 268: 87-99

[17] Escalona-Morán M A et al 2015 Electrocardiogram classification using reservoir computing with logistic regression. IEEE J. Biomed. Health Inf. 19(3): 892-898

[18] Xiang L et al 2017 Deep auto-context convolutional neural networks for standard-dose pet image estimation from lowdose pet/mri. Neurocomputing 267: 406-416

[19] Dhungel N, Carneiro G and Bradley A P 2017 A deep learning approach for the analysis of masses in mammograms with minimal user intervention. Med. Image Anal. 37: 114-128

[20] Lu Y et al 2017 Identification of rice diseases using deep convolutional neural networks. Neurocomputing 267: 378-384

[21] Affonso C et al 2017 Deep learning for biological image classification. Expert Syst. Appl. 85: 114-122

[22] Liang L, Liu M and Sun W 2017 A deep learning approach to estimate chemically-treated collagenous tissue nonlinear anisotropic stress-strain responses from microscopy images. Acta Biomater. 63: 227-235

[23] Zheng Y J et al 2017 Airline passenger profiling based on fuzzy deep machine learning. IEEE Trans. Neural Netw. Learn. Syst. 28: 2911-2923

[24] Gopalakrishnan K et al 2017 Deep convolutional neural networks with transfer learning for computer vision-based data-driven pavement distress detection. Constr. Build. Mater. 157: 322-330

[25] Deng W et al 2017 Deep correlation feature learning for face verification in the wild. IEEE Signal Process. Lett. 24(12): 1877-1881

[26] Arcos-García Á et al 2017 Exploiting synergies of mobile mapping sensors and deep learning for traffic sign recognition systems. Expert Syst. Appl. 89: 286-295

[27] Chen L, Qu H and Zhao J 2018 Generalized Correntropy based deep learning in presence of non-Gaussian noises. Neurocomputing 278: 41-50

[28] Qi T et al 2017 Image-based action recognition using hintenhanced deep neural networks. Neurocomputing 267: 475-488

[29] Lun S X et al 2015 A novel model of leaky integrator echo state network for time-series prediction. Neurocomputing 159(1): 58-66

[30] Jaeger H 2010 The "echo state" approach to analysing and training recurrent neural networks - with an Erratum note. GMD Report 148, pp. 1-47 